\title{
Anterior Cervical Osteophytes as a Cause of Progressive Dysphagia
}

\author{
S. M. Papadopoulos, J. C. Chen, J. A. Feldenzer, M. N. Bucci, and J. E. McGillicuddy \\ Section of Neurosurgery, University of Michigan, Ann Arbor, Michigan, U.S.A.
}

\section{Summary}

An uncommon cause of progressive dysphagia may be large cervical osteophytes impinging upon the cervical esophagus. Dysphagia may be due to the mechanical mass effect of a large anterior osteophyte, trapping the esophagus opposite a fixed point such as the cricoid cartilage, or a local inflammatory reaction resulting in cricopharyngeal spasm. We report three patients with progressive dysphagia due to large anterior cervical osteophytes. All three patients were treated with anterior cervical approach with removal of the osteophytes without fusion. A review of the literature in addition to the specific case histories, video fluoroscopic and radiographic findings are presented.

Keywords: Cervical osteophytes; dysphagia.

\section{Introduction}

An uncommon cause of progressive dysphagia may be large anterior cervical osteophytes impinging upon the cervical esophagus. While this has been recognized and described in the otolaryngologic $1,3,4,13,15-17,20$ literature, these cases have rarely been documented in the neurosurgical ${ }^{5,9}$ literature. In the past year, three patients with progressive dysphagia due to large anterior cervical osteophytes have been treated. The symptoms were severe enough to result in changes in dietary habits and ultimate weight loss in all three patients. All three patients were treated with an anterior cervical approach with removal of the spurs without entering the disc space and without fusion.

\section{Case Histories}

Case 1: This 65-year-old white male had a five year history of dysphagia and aspiration of fluids. He denies any symptoms suggestive of cervical radiculopathy or myelopathy. He also denies symptoms of neck pain or discomfort. Over the past one year he had altered his diet to include only soft foods and liquids and he had an approximately twenty pound weight loss over that period of time. Cervical spine filsm showed a very large C2-3 anterior cervical osteophyte in addition to a smaller osteophyte at C3-4 (Fig. 1).
Video fluroscopic swallowing study was notable for a constriction of contrast material at the level of the C2-3 anterior cervical osteophyte with associated vallecular pooling and laryngeal aspiration (Fig. 2). The patient underwent uneventful operative excision of the anterior cervical spurs through a routine anterior-lateral approach. He had marked improvement in swallowing function and was able to resume a normal diet. Postoperative cervical spine films showed absence of the cervical osteophytes (Fig. 3). There was no evidence of cervical instability postoperatively, either clinically or on routine X-rays. Additionally, postoperative video fluroscopy showed essentially a normal swallowing study (Fig. 4).

Case 2: A 64-year-old black woman presented with a thirty pound weight loss and a history of difficulty swallowing for one and

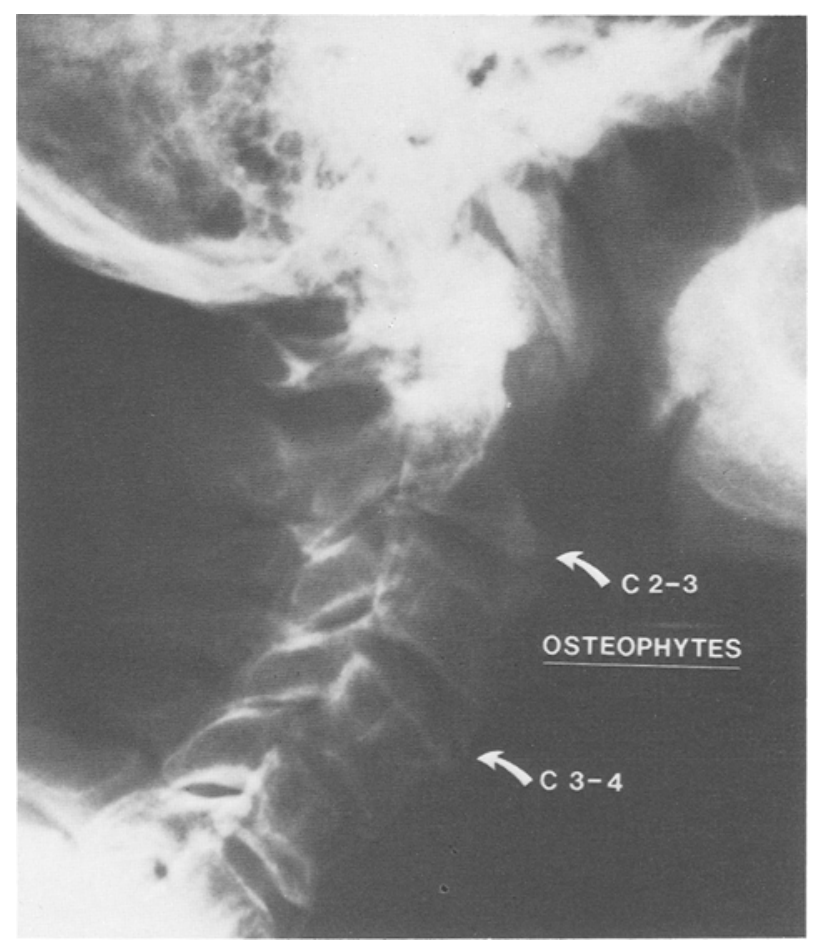

Fig. 1. A large C 2-3 and smaller C 3-4 anterior cervical osteophyte seen on the lateral cervical spine film 


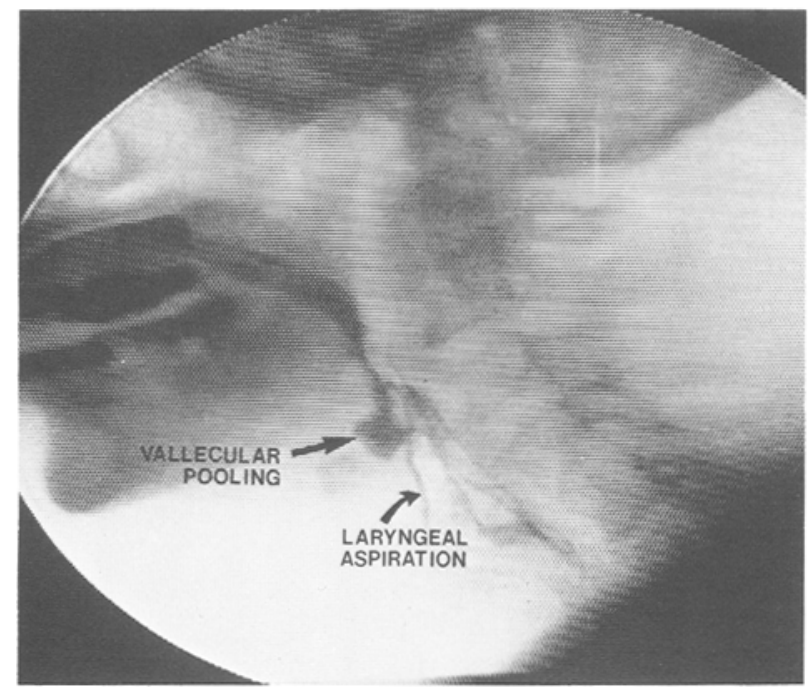

Fig. 2. Video fluoroscopic swallowing studies showing constriction of contrast material at the level of the C 2-3 osteophyte with associated vallecular pooling and laryngeal aspiration

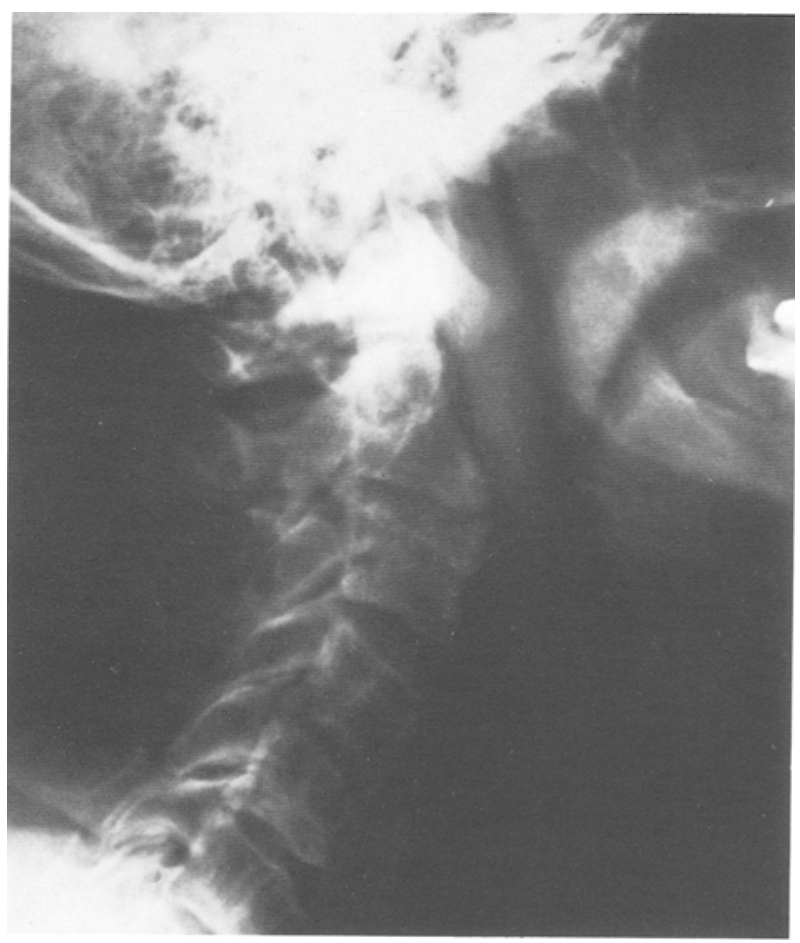

Fig. 3. Postoperative lateral cervical spine films shows absence of the previous $\mathrm{C} 2-3$ and $\mathrm{C} 3-4$ osteophytes

a half years. She had altered her diet to include only pureed foods, Jello and liquids. She had frequent episodes of aspiration and was often unable to clear her own salivary secretions. She denied any neck pain or symptoms suggestive of cervical radiculopathy or myelopathy. Investigation for occult malignancy was negative; however, cervical spine films did reveal anterior cervical osteophytes at $\mathrm{C} 4$ 5 and C 5-6. Video fluoroscopic swallowing study showed narrowing

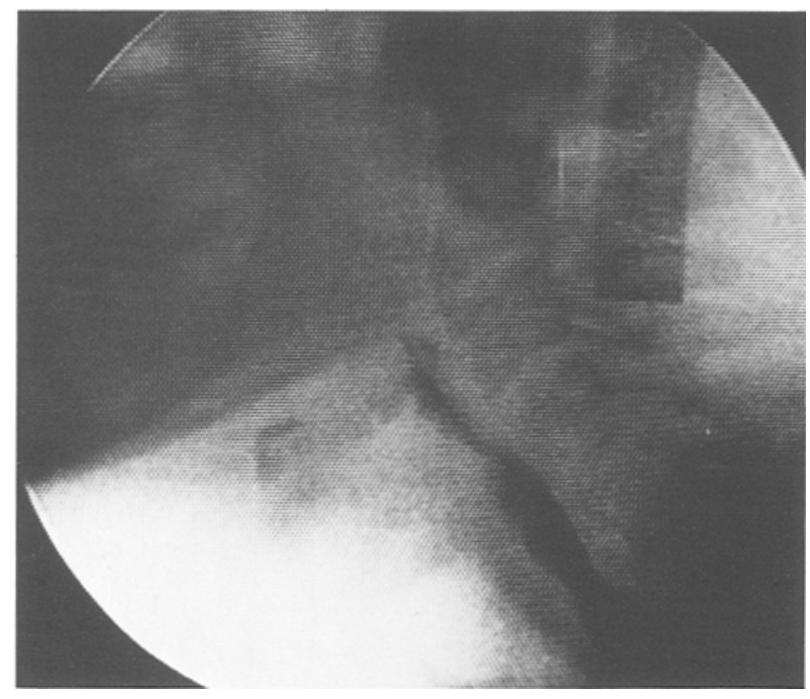

Fig. 4. Postoperative video fluoroscopy no longer shows constriction of contrast material, vallecular pooling or laryngeal aspiration

and pooling predominantly at the level of the C 5-6 spur. She underwent anterior cervical removal of the spurs at both the C4-5 and C 5-6 levels with difficulty. Postoperatively the patient's swallowing improved clinically with resultant weight gain over the next several months.

Case 3: A 54-year-old white male had difficulty with swallowing for the past eight years. This had become quite severe over the recent six months, requiring him to adjust his diet to include only soft foods, pureed meats, Jello and liquids. Cervical spine films showed a large anterior spur at C5-6. The swallowing study showed esophageal narrowing and pooling at this level. He underwent anterior cervical removal of the spurs without fusion. The disc space was not entered. Postoperatively he did extremely well and was able to resume a normal diet within days.

\section{Discussion}

In 1926, Mosher first described difficulty in swallowing caused by exostosis of the cervical vertebrae ${ }^{16}$. Asymptomatic hypertrophic spurs of the anterior cervical spine may occur in 20 to 30 per cent of the population ${ }^{4}$ 9. Large anterior osteophytes may also be found in conditions such as diffuse idiopathic skeletal hyperostosis (DISH) ${ }^{9-11}$, ankylosing spondolytis ${ }^{7}$, infectious spondolytis ${ }^{5}$ and following trauma ${ }^{9}$. The most frequent level of involvement related to dysphagia is C 5-6 followed by $\mathrm{C} 4-5, \mathrm{C} 2-3$ being the least common level affected $^{1,2,9,14}$. Enlarged anterior cervical osteophytes may cause dysphagia through several proposed mechanism. Mechanical distortion of the esophagus may occur due to the mass effect of a large anterior osteophyte. Osteophytes may also cause dysphagia if they are located opposite a fixed point of the esophagus such as the cricoid cartilage (C 6 level) ${ }^{9}$. Additionally, 
osteophytes may cause an inflammatory reaction around the esophagus resulting in edema and cricopharyngeal spasm. This has led a number of authors to suggest treatment with a trial of steroids prior to operative removal of the osteophytes ${ }^{4,10}$. The rare patient with dysphonia due to osteophytic disease may have vocal cord edema or recurrent laryngeal nerve injury ${ }^{5}$.

This condition occurs more frequently in men than women, typically in their 50 's and 60 's ${ }^{14}$. The symptomatic levels correspond to the incidence previously mentioned. The typical history consists of a long period of difficulty swallowing and a non-descript sensation of a lump in the throat. In progressive dysphagia, this may be followed by a shorter history of inability to swallow solid foods, aspiration and weight loss. Evaluation of patients with progressive dypshagia should include routine otolaryngologic examination, cervical spine films, video fluroscopic swallowing study and esophogoscopy, if indicated. It should be noted that esophagoscopy in the presence of large anterior cervical osteophytes present a significant risk of esophageal injury ${ }^{19}$. Large osteophytes also present a risk of esophageal injury during the operative exposure. The esophagus may be difficult to mobilize and somewhat adherent to other anterior cervical fascia due to local inflammatory reaction. Differential diagnosis includes esophageal tumors, esophageal stricture, Zenker's diverticulum, motility disorders, PlummerVincent's syndrome and other mediastinal mass lesions ${ }^{5,9,11}$. Additionally, ossification of the anterior longitudinal ligament, ankylosing spondylytis ${ }^{7,9}$. Atlantoaxial dislocation ${ }^{5}$, osteochondroma ${ }^{9}$, congenital bone bars ${ }^{11}$ and frank anterior disc herniation may cause dysphagia.

Non-surgical treatment options may include steroids and dietary modification. However, for progressive dysphagia, surgical excision of the anterior cervical osteophytes is recommended. Concomitant cervical fusion or diskectomy are not felt to be necessary. Reossification with new osteophyte formation may rarely occur and repeat operation may be indicated if dysphagia symptoms return ${ }^{12}$.

Hypertrophic anterior cervical osteophytes are an uncommon etiology of dysphagia. Anterior cervical excision of these osteophytes typically leads to excellent symptomatic results.

\section{References}

1. Bauer F (1953) Dysphagia due to cervical spondylosis. J Laryngol Otol 67: 615-630

2. Beahrs OH, Schmidt HW (1959) Dysphagia caused by hypertrophic changes in the cervical spine: Report of two cases. Ann Surg 149: 297-299

3. Blumberg P, Prapote C, Viscomi G (1977) Cervical osteophytes producing dysphagia. Ear Nose Throat J 56: 396-399

4. Bone RC, Nahum AM, Harris AS (1978) Evaluation and correction of dysphagia-producing cervical osteophytosis. Laryngoscope 84: 2045-2050

5. Brandenberg G, Leibrock LG (1986) Dysphagia and dysphonia secondary to anterior cervical osteophytes. Neurosurg 18:9093

6. Brooks AL, Ochsner SF (1964) Dysphagia caused by cervical osteophytes. Am J Orthop 6: 188-190

7. Carlson MJ, Stauffer RN, Payne WS (1974) Anklyosing vertebral hyperostosis causing dysphagia. Arch Surg 109: 567-570

8. Coventry MB (1970) Calcification in a cervical disc with anterior protrusion and dysphagia: A case report. J Bone Joint Surg (Am) 52A: 1463-1466

9. Gamache FW Jr, Voorhies RM (1980) Hypertrophic cervical osteophytes causing dysphagia: A review. J Neurosurg 53: 338344

10. Gohel VK, Karasick S, Canino C (1976) Cervical spondylotic dysphagia. JAMA 235: 935-936

11. Hilding DA, Tachdjian MO (1960) Dysphagia and bypertrophic spurring of the cervical spine. N Eng J Med 263: 11-14

12. Hirano H, Suzuki H, Sakakibara T, Higuchi $Y$, Inoue K, Suzuki Y (1982) Dysphagia due to hypertrophic cervical osteophytes. Clin Orthop 167: 168-172

13. Khalifa MC, Amr F, Fouly SE, Aouf M (1981) Ossification of the anterior longitudinal ligament of the spine as a cause of dysphagia. J Laryngol Otol 95: 527-528

14. Lambert JR, Tepperman PS, Jimenez J, Newman A (1981) Cervical spine disease and dysphagia: Four new cases and a review of the literature. Am J Gastroenterol 76: 35-40

15. Maran A, Jacobson I (1971) Cervical osteophytes presenting with pharyngeal symptoms. Laryngoscope 81 : 412-417

16. Mosher HP (1926) Exostoses of the cervical vertebrae as a cause for difficulty in swallowing. Laryngoscope 36: 181-182

17. Orton HB (1926) Anterior dislocation of the atlas as a cause for inability to swallow solid foods. Laryngoscope 36: 188-189

18. Ruffin J (1950) Dysphagia caused by exostoses of the cervical spine. Gastroenterology 16:589-592

19. Smith EEK, Tanner NC (1956) The complications of gastroscopy and oesophogoscopy. Br J Surg 43: 396-403

20. Umerah BC, Mukherjee BK, Ibedwe O (1981) Cervical spondylosis and dysphagia. J Laryngol Otol 95: 1179-1183

Correspondence and Reprints: Stephen M. Papadopoulos, M.D., Section of Neurosurgery, 1500 E. Medical Center Dr., Ann Arbor, Michigan 48109-0338, U.S.A. 